\title{
Properties of the Social Discount Rate in a Benthamite Framework with Heterogeneous Degrees of Impatience
}

\author{
Diego Nocetti \\ School of Business, Clarkson University, Potsdam, New York 13699, dnocetti@clarkson.edu \\ Elyès Jouini \\ Ceremade, Université Paris-Dauphine, F-75016 Paris, France, Jouini@ceremade.dauphine.fr \\ Clotilde Napp \\ CNRS, UMR7088, F-75016 Paris, France \\ DRM, Université Paris-Dauphine, F-75016 Paris, France, clotilde.napp@dauphine.fr
}

\begin{abstract}
This paper derives the properties of the discount rate that should be applied to a public-sector project when the affected population has heterogeneous degrees of impatience. We show that, for any distribution of discount rates, the social discount rate has the following properties: it decreases over time, it is lower than the average of the discount rates in the population and, it converges to the discount rate of the most patient individual in the economy. These properties hold for both constant and decreasing individual discount rates. Finally, we evaluate how changes in the distribution of individual discount rates affect the social discount rate.
\end{abstract}

Keywords: social discount rate; hyperbolic discounting; cost-benefit analysis

\section{Introduction}

The appropriate social discount rate (SDR) to apply in public-sector cost-benefit analyses is a contentious issue. As Weitzman (2001 p.261) states "There does not now exist, nor has ever existed, anything remotely resembling a consensus, even -or, perhaps one should say especially- among the 'experts' on this subject." Especially when the discount rates of the affected population are 
heterogeneous, social planners face the difficult problem of what weight to give to them. Of course, a straightforward solution would be to use a simple average of the discount rates. Although frequently applied in practice (Bazerlon and Smetters 1999), this method is, in general, incorrect. To see this, consider a project that provides a payoff of $\$ 200$ in 50 years to two individuals ( $\$ 100$ each), Patience who has a discount rate of one percent and Impatience who has a discount rate of ten percent. Using the average of the discount rates (5.5 percent) the present value of the project would be $\$ 12.8$. However, the present value of $\$ 100$ is about $\$ 61$ for Patience and $\$ 0.7$ for Impatience, for a total present value of the project of $\$ 61.7$ (which implies an SDR of about 2.4 percent). In this example, the average discount rate understates the present value of the project by a factor of about five.

Further complicating the aggregation of preferences, empirical evidence has shown systematic departures from the standard model in which all benefits and costs are discounted at the same rate. For instance, people discount outcomes in the near future at a higher rate than they do for more distant outcomes, i.e. hyperbolic discounting (Ainslie 1992, Cropper et al 1994). In addition, the discount rate may depend on the 'sign' (i.e. whether it is a cost or a benefit) and on the magnitude of the outcomes, with higher discount rates for gains (benefits) and small outcomes (Thaler 1981, Benzion et al 1989). ${ }^{1}$

Given these and other difficulties, it is not surprising that in practice government agencies use a wide variety of discount rates, ranging from zero to infinite percent, with little justification (Bazerlon and Smetters 1999). The aim of this paper is to provide some guiding principles for the selection of the appropriate SDR. Specifically, we consider a social planner who gathers data on individual discount rates, which may be constant or decreasing with time, and uses a Benthamite social welfare function to aggregate them. Then, we ask: what are the properties of the SDR that she will obtain?

This problem has been analyzed before in the literature by assuming specific distributions of the individual discount rates across the affected population. For example, Reinschmidt (2002) showed that if

\footnotetext{
${ }^{1}$ Frederick et al. (2002) present a review of the literature on hyperbolic discounting and other 'anomalies' usually observed in experimental studies of intertemporal choice.
} 
the individual discount rates are constant over time and normally distributed over the population, the SDR should be lower than the average of the individual rates and decreasing in time. In particular, suppose that a public project provides aggregate net benefits at time $t$ of $A_{t}$ and that the net benefits are divided equally among a population of size $n$. Further, suppose that individuals discount the net benefits with an exponential discount function, with heterogeneous discount rates $\rho_{i}$. Then, the present value of the benefits received at time $t$ is $A_{t} \frac{1}{n} \sum_{i} e^{-\rho_{i} t}$. For large $n$ we can write this as $A_{t} E e^{-\rho t}$, where $E$ is the expectations operator over the distribution of discount rates. Assuming that the discount rates are sampled from a normal distribution (with a mean discount $u$ and a variance $\sigma^{2}$ ), $e^{-\rho t}$ has a lognormal distribution and $E e^{-\rho t}=e^{-t\left(E \rho-t \frac{1}{2} \operatorname{Var}(\rho)\right)}=e^{-t\left(\mu-t \frac{1}{2} \sigma^{2}\right)}$. It follows that the SDR is $\rho_{\text {soc }}=\mu-\sigma^{2} t / 2$.

Based on this result, Reinschmidt (2002, p.311) argues that "The aggregate social discount rate $\rho(t)=\mu-\sigma^{2} t / 2$ which decreases with time and variance, can be used for evaluating public-sector projects even when individual discount rates are considered to be constant in time." Two linked problems arise from this suggestion. First, a crucial assumption of the model (that some individuals in the population have constant and extremely large negative discount rates) seems insufficiently plausible to justify placing serious reliance on the derived SDR. Second, and more important, the SDR decreases linearly with the project's horizon and, as a result, is negative for sufficiently long horizons. Although declining discounts seem to be an empirical regularity in public-sector projects (e.g., Henderson and Bateman, 1995, Bazerlon and Smetters 1999), a discount rate that goes to negative infinity as the project's horizon lengthens makes little sense. In order to deal with this issue Reinschmidt proposes: "To assure that $\rho(t) \geq 0$, Equation (14) -the SDR- is used only in the range $0 \leq t \leq 2 \mu / \sigma^{2}$." Therefore, for example, if $2 \mu / \sigma^{2}=20$, a project that provides benefits or costs beyond twenty periods should not utilize this discount rate and should instead use something else. One is left to wonder what that different discount rate should be (e.g., what rate should be applied to discount climate change damages?). 
Not only is constraining the time difficult to justify on any grounds, but also, the large heterogeneity in discount rates usually found in the data limits the applicability of the derived SDR to analyze any kind of project. For instance, in the fairly homogeneous population (economics and finance students) analyzed by Benzion et al. (1989), across the multiple treatments $2 \mu / \sigma^{2}$ was never larger that twenty and usually much smaller.

Henderson and Langford (1998) and Weitzman (2001) show that a hyperbolic social discounting function can also be obtained by assuming a gamma distribution of the individual discount rates. ${ }^{2}$ Yet, although the gamma distribution is a more sensitive choice to model the distribution of individual rates, an important question remains: is hyperbolic discounting a general property of social discounting when the affected population has heterogeneous discount rates? The same question regards the other properties derived (e.g. the SDR decreases with the variance of the individual discount rates). The main contribution of this paper is to characterize the properties of the SDR for any distribution of discount rates.

\section{General properties of the SDR}

Imagine a decision maker who gathers data on individual discount rates and needs to evaluate the present value of a project providing a stream of net benefits $A_{t}$ for $\mathrm{t}=0, \ldots, \mathrm{T}$. Consider a continuous population of measure one and suppose that fraction $x_{i}$ of the population (group $i$ ) has a constant and

\footnotetext{
${ }^{2}$ To be more precise, in Weitzman's (2001) paper the underlying heterogeneity is among the recommendations of a group of experts that a government agency consults.

${ }^{3}$ A declining SDR might also be justified if there is uncertainty about future discount rates (e.g. Newell and Pizer 2003) or about the state of the economy (e.g., Gollier 2002). In a quite general setting with heterogeneous individual discounts, Gollier and Zeckhauser (2005) relate the term structure of the social discount rate with the shape of the individual utility functions.
} 
non-negative discount rate $\rho_{i} \in\left[\rho_{\min } \geq 0, \rho_{\max }\right]$ (alternatively, $x_{i}$ can be interpreted as the proportion of $A_{t}$ that group $i$ receives) ${ }^{4}$

The present value of the stream of net benefits

$$
P=\sum_{t=0}^{T} A_{t} \sum_{i} x_{i} e^{-\rho_{i} t} .
$$

The social discount factor $e^{-h(t)}$ must satisfy, for every stream of net benefits $\left(A_{t}\right)_{t=0, \ldots, T}$ and for every horizon $T$

$$
\sum_{t=0}^{T} A_{t} e^{-h(t)}=\sum_{t=0}^{T} A_{t} \sum_{i} x_{i} e^{-\rho_{i} t}
$$

Since (1') must always hold, it follows that we must also have

$$
e^{-h(t)}=\sum_{i} x_{i} e^{-\rho_{i} t}
$$

As in Weitzman $(1998,2001)$, we define the (instantaneous) SDR as

$$
\rho_{s o c}=h^{\prime}(t)=\frac{\sum_{i} \rho_{i} x_{i} e^{-\rho_{i} t}}{\sum_{i} x_{i} e^{-\rho_{i} t}},
$$

which is a weighted mean of the individual discount rates.

An alternative to this 'marginal' SDR would be to consider the 'average' SDR, defined as $\rho_{a}=h(t) / t=-1 / t \ln \left(\sum_{i} x_{i} e^{-\rho_{i} t}\right)$. The relationship between the 'marginal' and the 'average' social discount rates is given by $\rho_{a}=1 / t \int_{0}^{t} \rho_{s o c}(s) d s$. We choose to analyze the properties of the 'marginal'

\footnotetext{
${ }^{4}$ The results that follow do not depend on the assumption that the individual discount rates are non-negative. Yet, as we show in Proposition 1, this restriction guarantees that the SDR applied to very long-term payoffs is non-negative.
} 
discount rate since it is easy to verify that all the properties of $\rho_{\text {soc }}$ presented in the paper carry over to $\rho_{a}$ (the converse is not true).

The following proposition characterizes the properties of $\rho_{s o c}$.

PROPOSITION 1. For any bounded distribution of the individual/group discount rates, the SDR is decreasing in t, it converges to $\rho_{\min }$ as the horizon lengthens, and it is lower than the weighted average of the different groups' discount rates, (i.e. $\rho_{\text {soc }}<\sum x_{i} \rho_{i}$ ).

Although we relegate all formal proofs to a web appendix, the intuition is simple. As a payoff is delayed, its present value decreases more rapidly for the more impatient groups; therefore, the relative weights in the SDR of the more impatient people decrease with time. As a result, the social planner will use a lower SDR for net benefits that are further in the future and for benefits received in the very long-term she will only use the discount rate of the most patient group (since they are the only ones that give some value to the project). ${ }^{5}$ Furthermore, because the individual discount functions are convex in the discount rate, the appropriate SDR to use is lower than the case when the social planner simply averages the different groups' discount rates.

We also want to consider whether the properties derived above hold if, as suggested by experimental evidence from multiple fields, individual discount rates decline with the time horizon. Specifically, suppose that group $i$ has a discount function of the form $D_{i}(t)=e^{-h_{i}(t)} \cdot{ }^{6}$ It follows that the discount rate is $\rho_{i}(t)=h_{i}^{\prime}(t)$, which obviously declines with time if $h_{i}^{\prime \prime}(t)<0$ (i.e. $h_{i}(t)$ is concave in $\left.t\right)$. The SDR is, as before, a weighted average of the groups' discount rates:

\footnotetext{
${ }^{5}$ A similar result is obtained in a quite different setting in Weitzman (1998). In fact, this is also the reason why Reinschmidt (2002)'s social discount rate tends to negative infinity, since in the case of a normal distribution the most patient individual has a constant discount rate of negative infinity.

${ }^{6}$ The exponential form is assumed here for ease of comparison with the case of constant discount rates; the only requirement to obtain the results, however, is that the discount function is convex in time.
} 


$$
\rho_{s o c}=\frac{\sum_{i} x_{i} h_{i}^{\prime}(t) e^{-h_{i}(t)}}{\sum_{i} x_{i} e^{-h_{i}(t)}} ;
$$

in fact, the SDR with constant discount rates is just a special case in which $h_{i}(t)=\rho_{i} t$ and $h_{i}^{\prime}=\rho_{i}$.

What are the properties of the SDR when the individual discount rates decline with the project's horizon? As one would expect, in addition to aggregation issues that make social discounting hyperbolic even when individual discount rates are constant, the fact that individuals become 'more patient' over time gives an additional reason for the SDR to decline over time. Also, as long as the discount function is convex, the appropriate discount factor for a given horizon will be higher than that applied by a social planner who uses the average of the groups' discount rates in the same discount function. Finally, the SDR converges to the lower bound of the support of the discount rates' distribution that holds in the limit. The intuition of this last result is as follows. When discount rates are constant, the weight that a given group receives in the SDR increases monotonically relative to the weights of the more impatient groups. Instead, when discount rates vary over time, patience is defined relative to the payoff's horizon; therefore, the relative weights may be non-monotonic. For instance, suppose that group 'A' discounts the near future more heavily than group 'B', but the discount rate of the former declines to a lower limiting level. Then, group A's relative weight will decline initially and, as they become more patient, it will eventually start to increase. In fact, the weight attached to the group with the lowest discount rate will converge to one.

In addition to establishing the properties of the SDR for a given population, we can evaluate how changes in the distribution of discount rates affect the SDR. Specifically, we want to determine conditions under which the SDR will unambiguously increase or decrease given a shift in the distribution of individual discount rates.

We begin by noting that, although first and second order stochastically dominated shifts in the distribution of discount rates increase the present value of the project, they are neither necessary nor 
sufficient to decrease the SDR for all horizons. Therefore, we need stronger notions of stochastic dominance.

DEFINITION 1. Monotone likelihood ratio dominance (MLR). Let $Y$ and $Z$ denote two random variables with respective distribution functions $F_{Y}$ and $F_{Z}$. We say that $Z$ dominates $Y$ in the sense of $\operatorname{MLR}\left(Z \succeq_{M L R} Y\right)$ if there exist numbers $-\infty \leq a \leq b \leq \infty$ and a non-decreasing function $h:[a, b] \rightarrow[0, \infty]$ such that $\operatorname{Pr}(Z<a)=0, \operatorname{Pr}(Y>b)=0$ and $d F_{Z}(x)=h(x) d F_{Y}(x)$ on $[a, b]$.

In other words, a MLR dominated shift for a given distribution puts less weight for higher values of $\rho$. When $Y$ and $Z$ have a finite common support $\rho_{1}<\ldots \ldots<\rho_{n}$ with respective weights $\left(x_{i}^{Y}\right)_{i=1, \ldots, n}$ and $\left(x_{i}^{Z}\right)_{i=1, \ldots, n}$ then $Z$ dominates $Y$ in the sense of MLR if and only if $\frac{x_{1}^{Z}}{x_{1}^{Y}} \leq \ldots . \leq \frac{x_{n}^{Z}}{x_{n}^{Y}}$. This concept is widely used in the statistical literature and was first introduced in the context of portfolio problems by Landsberger and Meilijson ${ }^{7}$ (1990). MLR dominance is stronger than FSD and, in particular, a MLR dominated shift for a given distribution reduces the mean.

DEFINITION 2. Portfolio Dominance (PD). Let $Y$ and $Z$ denote two random variables. We say that $Z$ dominates $Y$ in the sense of $P D\left(Z \succeq_{P D} Y\right)$ if we have $E\left[u^{\prime}(Z)(Z-a)\right]=0 \Rightarrow E\left[u^{\prime}(Y)(Y-a)\right] \leq 0$ for any real number $a$ and any non-decreasing and concave function $u$.

This concept has been introduced in the context of portfolio problems by Landsberger and Meilijson (1993) and further studied by Gollier (1997). It characterizes the changes in the distribution of the returns of the risky asset that lead to an increase in demand for the risky asset irrespective of the risk-free rate. In the portfolio context, it is then related to the degree of riskiness of the assets returns. In our context, it is related to individual discount rates' heterogeneity. In particular a mean preserving PD dominated shift for a given distribution increases the variance (Jouini and Napp, 2008).

\footnotetext{
${ }^{7}$ More precisely, Landsberger and Meilijson (1990) showed that in the standard portfolio problem a MLR shift in the distribution of returns of the risky asset leads to an increase in demand for the risky asset for all agents with nondecreasing utilities.
} 
This leads to

PROPOSITION 2. MLR and PD stochastically dominated shifts in the distribution of the individual discount rates reduce the SDR.

Therefore, although there is no necessary connection between the mean and the variance of the discount rates and the SDR (as in Reinschmidt 2002 or Weitzman 2001), there is a well defined sense in which projects in more patient and heterogeneous societies (in the sense of MLR and mean preserving PD shifts) should use smaller discounts. ${ }^{8}$ So, for instance, since discount rates are generally negatively correlated with wealth (e.g., Hausman 1979, Lawrance 1991) we should expect that, everything else constant, in countries with larger wealth inequality the discount rates applied to social projects will be lower. Of course, countries with high wealth inequality but also low wealth levels may still apply a relatively high SDR.

\section{Example}

To provide a simple example of what our results mean in practice, we calculate the SDR (Equation 2) using the frequency distribution of discount rates obtained by Weitzman (2001) in a survey of economists. ${ }^{9}$ Figure 1 illustrates the implied SDR for different horizons. Clearly, heterogeneity among the respondents in the survey makes the SDR decline substantially, from about 4 percent -the weighted mean in the sample- to 0 percent -the lowest discount rate-, and quite rapidly over the time horizon.

\footnotetext{
${ }^{8}$ For example, suppose that in one population (say "A") we have three equally large groups with discount rates $2 \%$, $4 \%$ and $6 \%$. In a second population ("B"), there are three groups with the same discount rates but with proportions in the population equal to $0.1,0.8$ and 0.1 respectively. It follows that both populations have the same weighted average of discount rates (4\%). However, because the distribution of discount rates in $\mathrm{A}$ is dominated in the sense of PD by the distribution of discount rates in $\mathrm{B}$, the discount rate for the former is lower for all $t$.

${ }^{9}$ The survey asked for the appropriate rate to discount long-term benefits and costs and not necessarily the respondents' rate of time preference. Our SDR was calculated 'as if' the response represented the individual's constant rate of time preference. See web appendix for further details.
} 
The SDR obtained is, for most time horizons, lower than the sequence of declining rates suggested by Moore et al. (2004). Of course, a different population may lead to different results. For instance, Proposition 2 states that in a more homogeneous population the appropriate SDR would be higher. Yet, we suspect that, for the United States as a whole, heterogeneity of discount rates is larger than in the sample of economists, which implies even lower not higher discount rates. Therefore, although our framework is not inconsistent with Moore et al. (2004), it does not provide strong support for the rates recommended.

Figure 1. SDR for Different Horizons

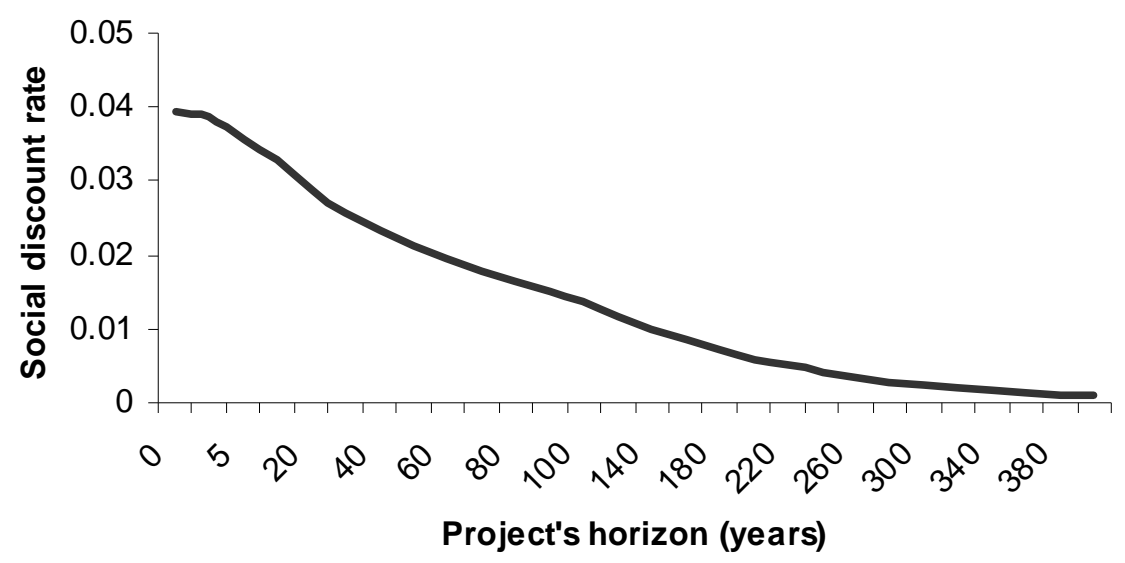

Note. We employed the frequency distribution of discount rates obtained by Weitzman (2001) [Table 1, p. 268] to calculate the proportion $x_{i}$ of the sample with a given discount rate $\rho_{i}$ in Equation (2). See web appendix for further details.

\section{Conclusions}

This paper provided a general characterization of the properties of the social discount rate when the discount rates of the affected population are heterogeneous and when using a Benthamite social welfare function. Importantly, we showed that the social discounting function should be hyperbolic when individual discount rates are declining or even constant. This is of great practical relevance since it implies that, for projects providing net benefits at different periods, decision-makers do not need to estimate the individual discount rates across time (a task that is likely to be very demanding) to justify 
applying declining discounts. For instance, Henderson and Langford (1998 p.1498-1499) state that "In practical terms the weight of evidence in favor of hyperbolic discounting must be particularly convincing if there is to be any likelihood of introducing its use in analyses by government agencies." Given the generality of our results, we believe that this paper provides such evidence.

Our results are particularly relevant for very long-term cost-benefit evaluations (e.g. those dealing with global climate change) that are especially sensitive to the selected discount rate. ${ }^{10}$ We showed that, for those cases, the appropriate SDR should be equal to the discount rate of the most patient individuals which, in practice, is likely to be close to zero percent.

\section{Acknowledgements}

This paper heavily relies on a previous version by D. Nocetti alone. The authors thank the associate editor and three anonymous referees for many helpful comments and suggestions. The financial support of the GIP-ANR ("Croyances" project) and of the Risk Foundation (Groupama chair) is gratefully acknowledged by Jouini and Napp.

\footnotetext{
${ }^{10}$ For instance, given the SDR estimated in the previous section, if one applies the short-term discount rate (4 percent) to a project that provides net benefits in say 300 years, the net benefits would be underestimated by a factor of about 80,000 (given an appropriate SDR of 0.24 percent).
} 


\section{References}

Ainslie, G.W. 1992. Picoeconomics. Cambridge: Cambridge University Press.

Bazerlon, C., K. Smetters. 1999. Discounting inside the Washington D.C. beltway. Journal of Economic Perspectives 13, 213-228.

Benzion, U., A. Rapoport, J. Yagel. 1989. Discount rates inferred from decisions: An experimental study. Management Science 35 (3), 270-284.

Cropper, M., S.K. Aydede, P.R. Portney. 1994. Preferences for life saving programs: How the public discounts time and age. Journal of Risk and Uncertainty 8, 243-265.

Frederick, S., G. Loewenstein, T. O'Donoghue. 2002. Time discounting and time preference: A critical review. Journal of Economic Literature 40(2), 351-401.

Gollier, C. 1997. A note on portfolio dominance. Review of Economic Studies 64, 147-150.

Gollier, C. 2002. Discounting an uncertain future. Journal of Public Economics 85, 149-166.

Gollier, C., R. Zeckhauser. 2005. Aggregation of Heterogeneous Time Preferences. Journal of Political Economy 113, 878-896.

Hausman, J.A. 1979. Individual discount rates and the purchase and utilization of energy-using durables. Bell Journal of Economics 10, 33-54.

Henderson, N., L. Bateman. 1995. Empirical and public choice evidence for hyperbolic social discounting and the implications for intergenerational discounting. Environmental and Resource Economics 5, 413-423.

Henderson, N., I. Langford. 1998. Cross-disciplinary evidence for hyperbolic social discount rates. Management Science 44 (11), 1493-1500.

Jouini E., C. Napp. 2008. On Abel's concept of doubt and pessimism. Journal of Economic Dynamics and Control. To appear.

Landsberger, M., I. Meilijson. 1990. Demand for risky financial assets: A portfolio analysis. Journal of Economic Theory 50, 204-213. 
Landsberger, M., I. Meilijson. 1993. Mean-preserving portfolio dominance. Review of Economic Studies 60, 479-485.

Lawrance, E.C. 1991. Poverty and the rate of time preference: Evidence from the panel data. Journal of Political Economy 99 (2), 54-77.

Moore, M., A. Boardman, A. Vining, D. Weimer, D. Greenberg. 2004. Just Give Me a Number: Practical Values for the Social Discount Rate. Journal of Policy Analysis and Management 23 (4), 789-812.

Newell, R., W. Pizer. 2003. Discounting the Benefits of Climate Change Mitigation: How Much do Uncertain Rates Increase Valuations? Journal of Environmental Economics and Management 46, $53-71$.

Reinschmidt, K.F. 2002. Aggregate social discount rate derived from individual discount rates. Management Science 48 (2), 307-312.

Thaler, R. H. 1981. Some empirical evidence on dynamic inconsistency. Economic Letters 8, 201-07.

Weitzman, M. 1998. Why the Far Distant Future Should be Discounted at its Lowest Possible Rate. Journal of Environmental Economics and Management 36, 201-208.

Weitzman, M. 2001. Gamma discounting. The American Economic Review 91 (1), 260-271. 


\section{Proofs of Statements and Data for Numerical Example}

\section{EC.1. PROOF OF PROPOSITION 1.}

PROPOSITION 1. For any bounded distribution of the individual/group discount rates, the SDR is decreasing in t, it converges to $\rho_{\min }$ as the horizon lengthens, and it is lower than the weighted average of the different groups' discount rates, (i.e. $\rho_{\text {soc }}<\sum x_{i} \rho_{i}$ ).

EC.1.1. Proof that $\partial \rho_{\text {soc }} / \partial t<0$

Denote $\theta_{i}=x_{i} e^{-\rho_{i} t}$. Taking the derivative of the SDR with respect to the project's horizon gives

$$
\frac{\partial \rho_{s o c}}{\partial t}=\frac{-\left(\sum_{i} \rho_{i}^{2} \theta_{i}\right) * \sum_{i} \theta_{i}+\left(\sum_{i} \rho_{i} \theta_{i}\right)^{2}}{\left(\sum_{i} \theta_{i}\right)^{2}}=-\left[\frac{\left(\sum_{i} \rho_{i}^{2} \theta_{i}\right)}{\left(\sum_{i} \theta_{i}\right)}-\left(\frac{\sum_{i} \rho_{i} \theta_{i}}{\sum_{i} \theta_{i}}\right)^{2}\right] .
$$

From Jensen's inequality we know that the second term in brackets must be equal or lower than the first term, proving the result that the sign of the derivative is non-positive (and negative when there is heterogeneity).

\section{EC.1.2. Proof that $\lim _{t \rightarrow \infty} \rho_{s o c}=\rho_{\min }$.}

Denote the (arbitrary) difference between the discount rate of the most patient group (with subscript $\min$ ) and any other group as $y_{i}$ (i.e., $\rho_{\min }=\rho_{i}-y_{i}$ ). Then, the SDR can be written as

$$
\begin{aligned}
\rho_{s o c}=\frac{\sum_{i} \rho_{i} x_{i} e^{-\rho_{i} t}}{\sum_{i} x_{i} e^{-\rho_{i} t}} & =\frac{\left(\rho_{\min } x_{\min } e^{-\rho_{\min } t}+\sum_{i \neq \min } \rho_{i} x_{i} e^{-\left(\rho_{\min }+y_{i}\right) t}\right)}{e^{-\rho_{\min } t}\left(x_{\min }+\sum_{i \neq \min } x_{i} e^{-y_{i} t}\right)} \\
& =z\left(\rho_{\min } x_{\min }+\sum_{i \neq \min } \rho_{i} x_{i} e^{-y_{i} t}\right),
\end{aligned}
$$


where $z \equiv\left(x_{\min }+\sum_{i \neq \min } x_{i} e^{-y_{i} t}\right)^{-1}$. Taking the limit as the project’s horizon goes to infinity yields, $\lim _{t \rightarrow \infty} z=x_{\min }^{-1}$ and $\lim _{t \rightarrow \infty} \sum_{i \neq \min } \rho_{i} x_{i} e^{-y_{i} t}=0$, which provides the result that $\lim _{t \rightarrow \infty} \rho_{s o c}=\rho_{\min }$.

EC.1.3. Proof that $\sum_{i} x_{i} e^{-\rho_{i} t} \geq \sum_{i} e^{-x_{i} \rho_{i} t}$

Again invoking Jensen's inequality, since $e^{-\rho_{i} t}$ is convex in $\rho_{i}$ we obtain that $\sum_{i} x_{i} e^{-\rho_{i} t} \geq \sum_{i} e^{-x_{i} \rho_{i} t}$, i.e., the appropriate discount factor (rate) is higher (lower) than the case when the social planner simply averages the groups discount rates.

\section{EC.2. PROOF OF RESULTS FOR THE DISCOUNT FUNCTION $D_{i}(t)=e^{-h_{i}(t)}$}

In the text we mentioned that the SDR for the discount function $D_{i}(t)=e^{-h_{i}(t)}$ has three properties: it decreases with time, it converges to the lower bound of the support of the distribution of discount rates that holds in the limit, and it is lower than the average discount rate.

EC.2.1. Proof that $\partial \rho_{\text {soc }} / \partial t<0$

The derivative of the SDR with respect to the project's horizon is

$\frac{\partial \rho_{s o c}}{\partial t}=\frac{-\sum_{i} \theta_{i}\left(h_{i}^{\prime}\right)^{2}}{\sum_{i} \theta_{i}}+\frac{\sum_{i} \theta_{i} h_{i}^{\prime \prime}}{\sum_{i} \theta_{i}}+\left(\frac{\sum_{i} \theta_{i} h_{i}^{\prime}}{\sum_{i} \theta_{i}}\right)^{2}$,

where $\theta_{i}=x_{i} e^{-h_{i}}$ and all the terms may be functions of time.

The first and third terms are isomorphic to the terms obtained in the case of constant discount rates. As before, by Jensen's inequality the sum of these terms is non-positive. The second term is new and results from the fact that the groups' discount rates change over time. Given our assumption that the discount rates decrease over time (i.e. $\left.h_{i}^{\prime \prime}(t)<0\right)$ it must be the case that $\partial \rho_{s o c} / \partial t<0$. 


\section{EC.2.2. Proof that $\lim _{t \rightarrow \infty} \rho_{\text {soc }}=h_{\min }^{\prime}(\infty)$.}

Remember that the SDR is a weighted average of the different groups' discount rates. Denote the weight of the group with $h_{\min }^{\prime}(\infty)$ as

$$
z_{\min }=\frac{x_{\min } e^{-h_{\min }(t)}}{\sum_{i} x_{i} e^{-h_{i}(t)}}
$$

where we used the subscript $\min$ in the fraction $x$ and the function $h(t)$ to refer to this group (i.e. it does not mean, for instance, that $x_{\min }$ is the lowest $x_{i}$ ). Note that

$$
\frac{z_{\min }}{z_{i}}=\frac{x_{\min } e^{-h_{\min }(t)}}{x_{i} e^{-h_{j}(t)}}=\frac{x_{\min }}{x_{i}} e^{-\left(h_{\min }(t)-h_{i}(t)\right)}
$$

Differentiating this ratio with respect to time we obtain

$$
\frac{\partial z_{\min } / z_{i}}{\partial t}=-\frac{x_{\min }}{x_{i}}\left(h_{\min }^{\prime}(t)-h_{i}^{\prime}(t)\right) e^{-\left(h_{\min }(t)-h_{i}(t)\right)} .
$$

This says that, if discount rates are constant, the weights of the more patient groups relative to the more impatient groups would increase monotonically over time. Therefore, $\lim _{t \rightarrow \infty} z_{\min } / z_{i}=\infty \forall i \neq \min$, i.e., the most patient group would receive a weight of 1 (which provides an alternative proof to the second part of Proposition 1). When discount rates are not constant the ratio of the weights may be non-monotonic; yet, because by definition it will eventually be true that $h_{\min }^{\prime}(t)<h_{i}^{\prime}(t), z_{\min }$ will approach 1 as in the case with constant discount rates.

\section{EC.2.3. Proof that the SDR is lower that the weighted mean of discount rates}

As long as the discount function is convex, by Jensen's inequality the appropriate discount factor will be higher than that applied by a social planner that uses the average of the groups' discount rates in the same discount function, i.e., $\sum_{i} x_{i} D_{i}\left(t, \rho_{i}(t)\right) \geq D_{i}\left(t, \sum_{i} x_{i} \rho_{i}(t)\right)$. 


\section{EC.3. PROOF OF PROPOSITION 2}

PROPOSITION 2. MLR and PD stochastically dominated shifts in the distribution of the individual discount rates reduce the $S D R$.

Let us consider $Y$ and $Z$ two random variables with associated distribution functions $F_{Y}$ and $F_{Z}$ such that $Z \succcurlyeq_{M L R} Y$. Without loss of generality, we may assume that $Y$ and $Z$ have the same support. By definition, the social discount rates $\rho_{s o c}^{Z}$ and $\rho_{s o c}^{Y}$ respectively associated to $Z$ and $Y$ are given by $\rho_{s o c}^{Z}=\frac{E\left[Z e^{-Z t}\right]}{E\left[e^{-Z t}\right]} \quad$ and $\quad \rho_{s o c}^{Y}=\frac{E\left[Y e^{-Y t}\right]}{E\left[e^{-Y t}\right]} . \quad$ We $\quad$ have $\quad$ then $\rho_{s o c}^{Z}=\frac{\int \rho e^{-\rho t} d F_{Z}(\rho)}{\int e^{-\rho t} d F_{Z}(\rho)}=\frac{\int \rho e^{-\rho t} h(\rho) d F_{Y}(\rho)}{\int e^{-\rho t} h(\rho) d F_{Y}(\rho)}=\frac{E^{\exp }[Y h(Y)]}{E^{\exp }[h(Y)]}$ where $h$ is non-decreasing and where $E^{\exp }$ is the expectation operator associated to the probability whose density with respect to the initial probability is equal to $\frac{e^{-Y t}}{E\left[e^{-Y t}\right]}$. Since $h$ is non-decreasing, we have $\iint\left(y_{1}-y_{2}\right)\left(h\left(y_{1}\right)-h\left(y_{2}\right)\right) d v \otimes d v\left(y_{1}, y_{2}\right) \geq 0$ with respect to any probability measure $v$ on the real line. We have then $\int y h(y) d v(y) \geq\left(\int y d v(y)\right)\left(\int h(y) d v(y)\right)$ and in particular $E^{\exp }[Y h(Y)] \geq E^{\exp }[Y] E^{\exp }[h(Y)]$ which leads to $\rho_{s o c}^{Z} \geq \rho_{s o c}^{Y}$.

Let us assume now that $Z \succcurlyeq_{P D} Y$. We have $\rho_{s o c}^{Z}=\frac{E\left[Z e^{-Z t}\right]}{E\left[e^{-Z t}\right]}$ and then $E\left[u(Z)\left(Z-\rho_{s o c}^{Z}\right)\right]=0$ with $u(x)=-e^{-x t}$. By definition, this implies that $E\left[u^{\prime}(Y)\left(Y-\rho_{s o c}^{Z}\right)\right] \leq 0$ hence $\rho_{s o c}^{Z} \geq \rho_{s o c}^{Y}$.

\section{EC.4. DATA FOR NUMERICAL EXAMPLE}

To calculate the SDR in Figure 1 we used equation (2) in the text, $\rho_{s o c}=\sum_{i} \rho_{i} \theta_{i} / \sum_{i} x_{i} \theta_{i}$, together with the data collected by Weitzman (2001) in a survey of economists. In particular, he asked the question: 
"Taking all relevant considerations into account, what real interest rate do you think should be used to discount over time the (expected) benefits and (expected) costs of projects being proposed to mitigate the possible effects of global climate change?"

The frequency distribution is reproduced in the following table.

\begin{tabular}{|rrr|}
\hline Discount rate (\%) & Frequency $\boldsymbol{*}^{\mathbf{p}}$ & $\boldsymbol{\boldsymbol { x } _ { \boldsymbol { i } }}$ \\
\hline 0 & 46 & 0.0213 \\
1 & 236 & 0.1094 \\
2 & 454 & 0.2105 \\
3 & 427 & 0.1980 \\
4 & 362 & 0.1678 \\
5 & 227 & 0.1052 \\
6 & 136 & 0.0631 \\
7 & 71 & 0.0329 \\
8 & 44 & 0.0204 \\
9 & 28 & 0.0130 \\
10 & 44 & 0.0204 \\
11 & 15 & 0.0070 \\
12 & 25 & 0.0116 \\
13 & 12 & 0.0056 \\
14 & 5 & 0.0023 \\
15 & 8 & 0.0037 \\
16 & 3 & 0.0014 \\
17 & 2 & 0.0009 \\
18 & 3 & 0.0014 \\
19 & 1 & 0.0005 \\
20 & 4 & 0.0019 \\
25 & 2 & 0.0009 \\
26 & 1 & 0.0005 \\
27 & 1 & 0.0005 \\
\hline
\end{tabular}

*Source: Table 1 p.268 Weitzman (2001). Responses (3) with a negative discount rate were excluded. 